\title{
VORTEX INDUCED VIBRATIONS OF A PAIR OF CYLINDERS AT REYNOLDS NUMBER 1000
}

\author{
S. Mittal, V. Kumar \\ Department of Aerospace Engineering \\ Indian Institute of Technology \\ Kanpur, UP 208016 \\ India
}

\begin{abstract}
Vortex induced vibrations of two equal-sized cylinders in tandem and staggered arrangement placed in uniform incompressible flow is studied. A stabilized finite element formulation is utilized to solve the governing equations. The Reynolds number for these $2 \mathrm{D}$ simulations is 1000 . The cylinders are separated by 5.5 times the cylinder diameter in the streamwise direction. For the staggered arrangement, the cross-flow spacing between the two cylinders is 0.7 times the cylinder diameter. In this arrangement, the downstream cylinder lies in the wake of the upstream one and therefore experiences an unsteady inflow. The wake looses its temporal periodicity, beyond a few diameters downstream of the front cylinder. The upstream cylinder responds as an isolated single cylinder while the downstream one undergoes disorganized motion. Soft-lock-in is observed in almost all the cases.
\end{abstract}

\section{Introduction}

Vortex induced vibrations of a pair of cylinders placed in a uniform flow represent an extremely complex flow problem. Some of the significant parameters that affect the flow past cylinders and their vibrations are the Reynolds number, relative arrangement of the cylinders, ratio of vortex shedding frequency $\left(F_{o}\right)$ to the structural frequency $\left(F_{s}\right)$, structural damping, mass of the cylinders, their surface roughness and level of free-stream turbulence. The understanding of this fluid-structure interaction phenomena is useful in various engineering applications such as design and analysis of flows past tall towers, chimneys, heat exchangers and off-shore structures. Most of the investigations, that these authors are familiar with, are based on laboratory experiments.

Zdravkovich [1] conducted a detailed investigation of flow-induced oscillations of two cylinders in various arrangements. For each of the arrangement, the range of reduced velocity $\left(=1 / F_{s}\right)$ for which oscillations occur and the value for maximum amplitude was measured. The oscillation amplitudes of the two cylinders depend very strongly on their relative locations. The oscillations of one cylinder can affect the vortex shedding and synchronization (lock-in) of the other. It was observed that in some cases the displacement of the front cylinder is larger than the rear one while in most of the cases the rear cylinder suffers larger amplitude oscillations. He classified the response of cylinders in three categories: (a) instability builds up rapidly to large amplitude primarily in streamwise direction, (b) instability builds up slowly to a certain 
amplitude with oscillations mainly in streamwise direction and (c) instability grows gradually to large oscillation amplitudes primarily in cross-flow direction.

Jendrzejczyk et al. [2] have conducted a detailed study with cylinders in tandem and transverse arrangement with spacing as 1.5 and 1.75 times the cylinder diameter. They observed four modes of vibrations for the various cylinder arrangements, although different modes are dominant at different values of reduced flow velocity $\left(1 / F_{s}\right)$. They also observed that the fluid damping for the cross-flow vibrations vary with the vibration amplitude of the cylinders.

King and Johns [3] reported a detailed study for two flexible cylinders in tandem arrangement in water flow. The inter-cylinder spacing, in their investigation, varied from 1.5 to 7 times the cylinder diameter. In some of the experiments the cylinders are coupled to each other via a horizontal member. It is rigid in certain cases and flexible in the others. Three modes of vibrations were observed for different ranges of $F_{s}$ : fundamental mode in-line, fundamental mode cross-flow and second normal mode in-line. The choice of modes is sensitive to the Reynolds number. For in-line motion of the cylinders the Reynolds number must exceed $1200-1500$.

Tsui [4] has proposed a mathematical model to simulate the wake-induced flutter of a cylinder. However, like several other models proposed by various researchers in the literature, the modeling of fluid dynamic forces is quite simple and does not incorporate the dependence on cylinder motion and other related parameters. Flow past a pair of stationary cylinders, itself, is not very well understood at this point. Therefore, it is the belief of these authors that it is almost impossible to construct a general model that predicts the response of the cylinders in any arbitrary arrangement. Some of the other works on flow induced vibrations of two cylinders can be found in articles by Bokaian and Geoola [5], Knisely and Kawagoe [6], Matsumoto et al. [7] and Chen [8].

Fluid dynamic interaction between two cylinders even when they are stationary is quite complex and sensitive to their relative arrangement $[8,9]$. Some of the interesting phenomena associated with such flows include coupled vortex streets, intermittent vortex shedding and biased flow that may lead to flopping of the wake. Mittal et al. [10] reported results for a systematic study involving cylinders in tandem and staggered arrangements with various spacings for Reynolds numbers 100 and 1000. The wake is very organized at low Reynolds number and temporal periodicity is observed in all the cases that result in unsteady solutions. Two of the arrangements simulated by them exhibit wake interference. In one case the cylinders are arranged in tandem with their centers separated by 5.5 times the cylinder diameter. In the second case the spacing between the cylinders in the flow direction is same as before while the spacing in cross-flow direction is 0.7 times the cylinder diameter. In both the cases the upstream cylinder behaves very similar to an isolated single cylinder while the downstream one, that lies in the unsteady wake of the first one, experiences large unsteady forces. While the flow is temporally periodic in the near wake of the upstream cylinder the flow in the wake of the downstream cylinder does not exhibit such periodicity.

A fairly comprehensive computational study for the vortex-induced oscillations of a single cylinder placed in a uniform flow has been reported in articles by Mittal and Tezduyar [11] and Mittal and Kumar [12, 13] for $R e=325,1000,1500$ and $10^{4}$. Lock-in, is observed for a range of structural frequencies of the oscillator; the vortex-shedding frequency of an oscillating cylinder changes to the structural frequency [14]. For oscillators whose mass, compared to the mass of the surrounding fluid displaced by the cylinder, is low soft-lock-in is observed [12, 13]. The vortex-shedding frequency of the oscillating cylinder does not exactly match the structural frequency; there is a slight detuning. This is one of the mechanisms of the non-linear oscillator to self-limit its oscillation amplitude.

Hysteresis in the cylinder motion during increasing and decreasing flow speeds has been observed in experiments [15]. Mittal and Tezduyar [11] also observed the same in their compu- 
tational study. In certain cases the motion of the cylinder leads to modes of vortex shedding that are very different than the one usually observed for flow past a stationary cylinder. Sometimes, a competition between various modes of vortex shedding is observed. The choice of the mode(s) of vortex shedding is sensitive to the Reynolds number, oscillation amplitude and the ratio $F_{s} / F_{o}[16,17,18]$.

In this article computational results for flow-induced oscillations of two cylinders placed in uniform flow at $R e=1000$ in staggered and tandem arrangements are reported. The cylinders are separated by 5.5 times their diameter in the streamwise direction. For the case of staggered arrangement the cross-flow spacing between the two cylinders is 0.7 times the cylinder diameter. Both these arrangements correspond to the case of wake interference. Results for flow-induced oscillations at $R e=100$ were reported in an earlier article [19].

The outline of the rest of the article is as follows. A brief review of the governing equations for incompressible fluid flow and for the motion of a rigid body under the influence of unsteady fluid forces is given in Section 2. Section 3 describes the stabilized finite element formulation of the governing equations. It is based on the space-time finite element method in which the finite element interpolation functions depend, both, on space and time. This way the deformation of the spatial domain is taken into account automatically. This method, known as the DSD/SST (Deforming Spatial Domain/Stabilized Space-Time) technique, was introduced by Tezduyar et al. [20, 21]. Since then, it has been used for a variety of problems involving fluid-structure interactions and free-surfaces. To stabilize the computations against spurious numerical oscillations and to enable the use of equal-order-interpolation velocity-pressure elements the Galerkin/Least-Squares (GLS) stabilization technique is employed. Section 3 describes the finite element formulation incorporating these stabilizing terms. The non-linear equation systems resulting from the finite-element discretization of the flow equations are solved using the Generalized Minimal RESidual (GMRES) technique [22] in conjunction with diagonal preconditioners. Results and discussions are presented in Section 4 and we end with some concluding remarks in Section 5 .

\section{The governing equations}

Let $\Omega_{t} \subset \mathbb{R}^{n_{s d}}$ and $(0, T)$ be the spatial and temporal domains respectively, where $n_{s d}$ is the number of space dimensions, and let $\Gamma_{t}$ denote the boundary of $\Omega_{t}$. The spatial and temporal coordinates are denoted by $\mathbf{x}$ and $t$. The Navier-Stokes equations governing incompressible fluid flow are

$$
\begin{aligned}
& \rho\left(\frac{\partial \mathbf{u}}{\partial t}+\mathbf{u} \cdot \nabla \mathbf{u}-\mathbf{f}\right)-\boldsymbol{\nabla} \cdot \boldsymbol{\sigma}=0 \quad \text { on } \Omega_{t} \text { for }(0, T) \text {, } \\
& \boldsymbol{\nabla} \cdot \mathbf{u}=0 \quad \text { on } \Omega_{t} \text { for }(0, T) .
\end{aligned}
$$

Here $\rho, \mathbf{u}, \mathbf{f}$ and $\boldsymbol{\sigma}$ are the density, velocity, body force and the stress tensor, respectively. The stress tensor is written as the sum of its isotropic and deviatoric parts:

$$
\boldsymbol{\sigma}=-p \mathbf{I}+\mathbf{T}, \quad \mathbf{T}=2 \mu \boldsymbol{\varepsilon}(\mathbf{u}), \quad \boldsymbol{\varepsilon}(\mathbf{u})=\frac{1}{2}\left((\nabla \mathbf{u})+(\boldsymbol{\nabla u})^{T}\right)
$$

where $\mathrm{p}$ and $\mu$ are the pressure and coefficient of dynamic viscosity. Both the Dirichlet and Neumann-type boundary conditions are accounted for, represented as

$$
\mathbf{u}=\mathbf{g} \text { on }\left(\Gamma_{t}\right)_{g}, \quad \mathbf{n} \cdot \boldsymbol{\sigma}=\mathbf{h} \text { on }\left(\Gamma_{t}\right)_{h}
$$


where $\left(\Gamma_{t}\right)_{g}$ and $\left(\Gamma_{t}\right)_{h}$ are complementary subsets of the boundary $\Gamma_{t}$ and $\mathrm{n}$ is its unit normal vector. The initial condition on the velocity is specified on $\Omega_{t}$ at $t=0$ :

$$
\mathbf{u}(\mathbf{x}, 0)=\mathbf{u}_{0} \text { on } \Omega_{0},
$$

where $\mathbf{u}_{0}$ is divergence free. A solid body immersed in the fluid experiences unsteady forces and in certain cases may exhibit rigid body motion. The motion of the body, in the two directions along the cartesian axes, is governed by the following equations:

$$
\begin{aligned}
\ddot{X}+4 \pi F_{s} \zeta \dot{X}+\left(2 \pi F_{s}\right)^{2} X=\frac{C_{D}}{2 M} & \text { for }(0, T), \\
\ddot{Y}+4 \pi F_{s} \zeta \dot{Y}+\left(2 \pi F_{s}\right)^{2} Y=\frac{C_{L}}{2 M} & \text { for }(0, T) .
\end{aligned}
$$

Here, $F_{s}$ is the reduced natural frequency of the oscillator, $\zeta$ is the structural damping coefficient, $M$ is the non-dimensional mass of the body while $C_{L}$ and $C_{D}$ are the instantaneous lift and drag coefficients for the body, respectively. The free-stream flow is assumed to be along the x-axis. $\ddot{X}, \dot{X}$ and $X$ denote the normalized in-line acceleration, velocity and displacement of the body, respectively, while $\ddot{Y}, \dot{Y}$ and $Y$ represent the same quantities associated with the cross-flow motion. In the present study, in which the rigid body is a circular cylinder, the displacement and velocity are normalized by the diameter of the cylinder and the free-stream speed, respectively. The reduced natural frequency of the system, $F_{s}$ is defined as $\frac{2 f_{s} a}{U_{\infty}}$ where $f_{s}$ is the actual frequency of the oscillator, $a$ is the radius of the cylinder and $U_{\infty}$ is the free-stream speed of the flow. The non-dimensional mass of the cylinder is defined as $M=\frac{m_{b}}{\rho_{\infty} a^{2}}$ where $m_{b}$ is the actual mass of the oscillator per unit length and $\rho_{\infty}$ is the density of the fluid. It may also be expressed as $M=\pi \frac{\rho_{s}}{\rho_{\infty}}$ where, $\rho_{s}$ is the effective density of the material of the cylinder. The force coefficients are computed by carrying an integration, that involves the pressure and viscous stresses, around the circumference of the cylinder:

$$
\begin{aligned}
& C_{D}=\frac{1}{\frac{1}{2} \rho_{\infty} U_{\infty}^{2} 2 a} \int_{\Gamma_{c y l}}(\sigma \mathrm{n}) \cdot \mathrm{n}_{\mathrm{x}} d \Gamma \\
& C_{L}=\frac{1}{\frac{1}{2} \rho_{\infty} U_{\infty}^{2} 2 a} \int_{\Gamma_{c y l}}(\sigma \mathrm{n}) \cdot \mathrm{n}_{\mathrm{y}} d \Gamma .
\end{aligned}
$$

Here $n_{x}$ and $n_{y}$ are the cartesian components of the unit vector $n$ that is normal to the cylinder boundary $\Gamma_{c y l}$. These coefficients include the fluid dynamic damping and the added mass effect. The initial conditions for the displacement and velocity of the cylinder are specified at $t=0$ :

$$
\begin{gathered}
X(0)=X_{0}, \quad \dot{X}(0)=\dot{X}_{0}, \\
Y(0)=Y_{0}, \quad \dot{Y}(0)=\dot{Y}_{0} .
\end{gathered}
$$

The equations governing the fluid flow are solved in conjunction with those for the motion of each of the two cylinders. The force acting on the body is calculated by integrating the flow variables on the body surface. The resulting drag and lift coefficients are used to compute the displacement and velocity of the body which are then used to update the location of the body and the no-slip boundary condition for the velocity field on the body surface.

\section{Finite element formulation}

To accommodate the motion of the cylinder and the deformation of the mesh, a formulation that can handle moving boundaries and interfaces is employed. In order to construct the 
finite element function spaces for the space-time method, we partition the time interval $(0, T)$ into subintervals $I_{n}=\left(t_{n}, t_{n+1}\right)$, where $t_{n}$ and $t_{n+1}$ belong to an ordered series of time levels: $0=t_{0}<t_{1}<\cdots<t_{N}=T$. Let $\Omega_{n}=\Omega_{t_{n}}$ and $\Gamma_{n}=\Gamma_{t_{n}}$. We define the space-time slab $Q_{n}$ as the domain enclosed by the surfaces $\Omega_{n}, \Omega_{n+1}$, and $P_{n}$, where $P_{n}$ is the surface described by the boundary $\Gamma_{t}$ as $t$ traverses $I_{n}$. As is the case with $\Gamma_{t}$, the surface $P_{n}$ is decomposed into $\left(P_{n}\right)_{g}$ and $\left(P_{n}\right)_{h}$ with respect to the type of boundary condition (Dirichlet or Neumann) being imposed. For each space-time slab we define the corresponding finite element function spaces: $\left(\mathcal{S}_{\mathbf{u}}^{h}\right)_{n},\left(\mathcal{V}_{\mathbf{u}}^{h}\right)_{n},\left(\mathcal{S}_{p}^{h}\right)_{n}$, and $\left(\mathcal{V}_{p}^{h}\right)_{n}$. Over the element domain, this space is formed by using first-order polynomials in space and time. Globally, the interpolation functions are continuous in space but discontinuous in time.

The stabilized space-time formulation for deforming domains is then written as follows: given $\left(\mathbf{u}^{h}\right)_{n^{-}}$, find $\mathbf{u}^{h} \in\left(\mathcal{S}_{\mathbf{u}}^{h}\right)_{n}$ and $p^{h} \in\left(\mathcal{S}_{p}^{h}\right)_{n}$ such that $\forall \mathbf{w}^{h} \in\left(\mathcal{V}_{\mathbf{u}}^{h}\right)_{n}, q^{h} \in\left(\mathcal{V}_{p}^{h}\right)_{n}$,

$$
\begin{array}{r}
\int_{Q_{n}} \mathbf{w}^{h} \cdot \rho\left(\frac{\partial \mathbf{u}^{h}}{\partial t}+\mathbf{u}^{h} \cdot \boldsymbol{\nabla} \mathbf{u}^{h}-\mathbf{f}\right) d \Omega+\int_{Q_{n}} \boldsymbol{\varepsilon}\left(\mathbf{w}^{h}\right): \boldsymbol{\sigma}\left(p^{h}, \mathbf{u}^{h}\right) d Q+\int_{Q_{n}} q^{h} \boldsymbol{\nabla} \cdot \mathbf{u}^{h} d Q \\
+\sum_{e=1}^{n_{e l}} \int_{Q_{n}^{e}} \frac{1}{\rho} \tau\left[\rho\left(\frac{\partial \mathbf{w}^{h}}{\partial t}+\mathbf{u}^{h} \cdot \boldsymbol{\nabla} \mathbf{w}^{h}\right)-\boldsymbol{\nabla} \cdot \boldsymbol{\sigma}\left(q^{h}, \mathbf{w}^{h}\right)\right] . \\
\\
{\left[\rho\left(\frac{\partial \mathbf{u}^{h}}{\partial t}+\mathbf{u}^{h} \cdot \boldsymbol{\nabla} \mathbf{u}^{h}-\mathbf{f}\right)-\boldsymbol{\nabla} \cdot \boldsymbol{\sigma}\left(p^{h}, \mathbf{u}^{h}\right)\right] d Q} \\
+\sum_{e=1}^{n_{e l}} \int_{Q_{n}^{e}} \delta \boldsymbol{\nabla} \cdot \mathbf{w}^{h} \rho \boldsymbol{\nabla} \cdot \mathbf{u}^{h} d Q+\int_{\Omega_{n}}\left(\mathbf{w}^{h}\right)_{n}^{+} \cdot \rho\left(\left(\mathbf{u}^{h}\right)_{n}^{+}-\left(\mathbf{u}^{h}\right)_{n}^{-}\right) d \Omega=\int_{\left(P_{n}\right)_{h}} \mathbf{w}^{h} \cdot \mathbf{h}^{h} d P
\end{array}
$$

This process is applied sequentially to all the space-time slabs $Q_{0}, Q_{1}, \ldots, Q_{N-1}$. In the variational formulation given by Eq. (12), the following notation is being used:

$$
\begin{array}{r}
\left(\mathbf{u}^{h}\right)_{n}^{ \pm}=\lim _{\varepsilon \rightarrow 0} \mathbf{u}\left(t_{n} \pm \varepsilon\right), \\
\int_{Q_{n}}(\ldots) d Q=\int_{I_{n}} \int_{\Omega_{n}}(\ldots) d \Omega d t \\
\int_{P_{n}}(\ldots) d P=\int_{I_{n}} \int_{\Gamma_{n}}(\ldots) d \Gamma d t .
\end{array}
$$

The computations start with

$$
\left(\mathbf{u}^{h}\right)_{0}^{-}=\mathbf{u}_{0}
$$

where $\mathbf{u}_{0}$ is divergence free.

The variational formulation given by Eq. (12), includes certain stabilization terms added to the basic Galerkin formulation to enhance its numerical stability. Details on the formulation, including the definitions of the coefficients $\tau$ and $\delta$, can be found in the references $[20,21,23]$.

\section{Results and Discussion}

Two equal-sized cylinders, of unit radii, reside in a rectangular computational domain whose upstream and downstream boundaries are located at 5 and 30 cylinder diameters, respectively, from from the center of the upstream cylinder. The upper and lower boundaries are placed at 10 diameters, each, from the center of the first cylinder. The no-slip condition is satisfied for the velocity on the cylinder wall and free-stream values are assigned for the velocity at the upstream boundary. At the downstream boundary, we specify a Neumann type boundary condition for 
the velocity that corresponds to zero viscous stress vector. On the upper and lower boundaries, the component of velocity normal to and the component of stress vector along these boundaries is prescribed zero value. Computations are carried out for two different relative locations of the cylinders and for Reynolds number 1000. Computations for $R e=100$ were reported in an earlier article [19]. Reynolds number is based on the diameter of the cylinders, free-stream velocity and the viscosity of the fluid. The non dimensional distance between the centers of the two cylinders is denoted by $P / D$ in the flow direction and by $T / D$ in the cross-flow direction as shown in Figure 1. The quantities with suffix 1 are associated with upstream cylinder while those with suffix 2 are for the downstream one.

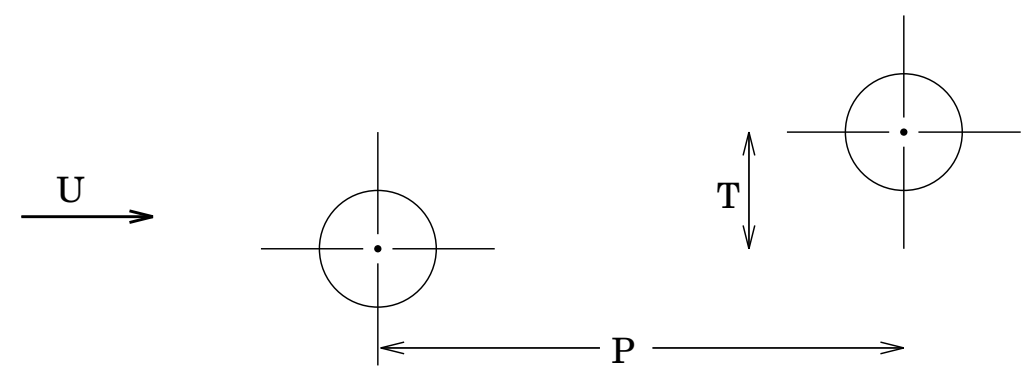

Figure 1: Description of the relative location of the two cylinders.

Computations are carried out for two sets of cylinder arrangements. In one case the two cylinders are arranged in tandem with $T / D=0$ and $P / D=5.5$ while the other case is that of staggered arrangement with $T / D=0.7$ and $P / D=5.5$. Results are presented for the vortexinduced oscillations of the two cylinders for various values of the structural frequency of the spring-mass oscillator $\left(F_{s}\right)$ that are either equal or close to the vortex-shedding frequency for the stationary cylinders $\left(F_{o}\right)$.

The calculations are carried out on a finite element mesh with 10, 432 nodes and 10, 168 elements. The mesh is similar to the one employed for the computations for $R e=100$ and reported in the article by Mittal and Kumar [19]. It has been designed to accommodate a meshmoving scheme that eliminates the need of re-meshing. Each of the two cylinders resides in a square box. The mesh within these boxes moves as rigid body along with the cylinders. The external boundary of the domain is fixed and the motion of the cylinders is accounted for by relocating the nodes outside the square boxes. With this mesh-moving scheme the connectivity of the mesh remains unaltered throughout the computations, thereby, eliminating the need of complex data-structure. Computations with a slightly coarser mesh with 8552 nodes and 8327 elements result in solutions that are different by less than $1 \%$ with those obtained with the original mesh. This establishes the adequacy of the present mesh that is being utilized for all the computations in this article.

The initial condition for all the computations is the fully developed unsteady solution past stationary cylinders in the same arrangement. The initial displacement and velocity of the two cylinders, in cross-flow and in-line directions, is assumed to be zero. Flows at $R e=1000$ are expected to have three-dimensional effects [24]. For example, it was reported in [10] that compared to experimental results and those from 3D calculations, 2D computations for $R e=1000$ flow past a stationary cylinder overpredict the mean drag coefficient and the Strouhal number related to the vortex-shedding frequency. However, due to restriction of computational resources, the computations reported in the present article are limited to 2D and it is hoped that they represent the true situation, at least, in an approximate sense. The non dimensional mass of each of the two cylinders is $M=4.7273$ and the structural damping coefficient is $\zeta=3.3 \times 10^{-4}$. These parameter values are same as those used for the studies involving single 
cylinder $[12,13]$.

\subsection{Flow past two cylinders in tandem arrangement; $T / D=0, P / D=5.5$}

Flow past two stationary cylinders in this arrangement at $R e=1000$ is temporally periodic in the near wake but quite disorganized in the wake of the downstream cylinder [12]. The time varying component of the aerodynamic forces acting on the downstream cylinder are significantly higher than those for the upstream one. The non dimensional value of the vortexshedding frequency is $F_{o}=0.234$ for both the cylinders. The vortex shedding from the two cylinders is almost antiphase.

\subsection{1 $\quad F_{s}=F_{o}$}

Figure 2 shows the time histories of the lift and drag coefficients and the response of the cylinders for $F_{s}=0.234\left(=F_{o}\right)$ for the fully developed solution. The upstream cylinder behaves like a single cylinder and its trajectory corresponds to a Lissajous figure of 8 . The downstream cylinder, however, exhibits low amplitude oscillations and does not reach a temporally periodic state. The non dimensional frequency corresponding to the cross-flow oscillations of both the cylinders is 0.226 . The lift coefficient for the upstream cylinder varies with a dominant non dimensional frequency of 0.226 while the dominant frequency for the downstream cylinder is 0.086. This suggests that soft-lock-in is observed only for the upstream cylinder at $R e=$ 1000 when $F_{s}=F_{o}$. This is in contrast to the observation for the $R e=100$ flow where the downstream cylinder lying in an unsteady but extremely organized wake of the upstream cylinder exhibits large amplitude oscillations.

Figure 2 also shows the pressure and vorticity fields for the fully developed solution. Unlike the $R e=100$ flow, two distinct vortex-streets are observed for the two cylinders and the flow exhibits temporal periodicity in the near wake of the first cylinder only. The vortex shedding mode of the upstream cylinder appears to be of the $2 \mathrm{P}$ type. One strong vortex is shed from the upper and lower surface of the cylinder, each, during every vortex shedding cycle. A closer look at the wake shows that each of the strong vortices is accompanied by a counter-rotating weak vortex that seems to suggest that the mode of vortex shedding is $2 \mathrm{P}[16]$.

\subsection{2 $\quad F_{s}=0.85 F_{o}$}

Figure 3 shows the time histories of the lift and drag coefficients and the response of the cylinders for $F_{s}=0.20\left(=0.85 F_{o}\right)$ for the fully developed solution. Unlike the previous case, the downstream cylinder in the present case oscillates with a fairly large amplitude. However its motion is not as organized as that of the upstream cylinder. The vortex-shedding frequency and the cross-flow oscillation frequency of the upstream cylinder is 0.205 . The dominant frequency in the time history for the variation of lift coefficient for the downstream cylinder is 0.140 while that for the cross-flow oscillation is 0.205 . As was observed for $F_{s}=F_{o}$, the downstream cylinder does not show lock-in. Shown in Figure 3 are the pressure and vorticity fields for the fully developed solution. Temporal periodicity of the solution is observed only in the near wake of the upstream cylinder. Even though the vortices shed from the first cylinder do not hit the second cylinder, they influence the vortex shedding from the downstream cylinder significantly. The vortices coalesce into big blobs as a result of intense mixing within a very short distance downstream of the second cylinder. It is interesting to note that the wake downstream of the second cylinder, in the present case, is even more disorganized than the one for $F_{s}=F_{o}$. Flowinduced oscillations of a single cylinder attain high amplitude for a range of values of $F_{s}$ that is usually centered around $F_{s}=F_{o}$. The present simulation suggests that, for the two cylinder 
arrangement, the maximum amplitude oscillations may not occur at $F_{s}=F_{o}$, but for reduced velocity $\left(1 / F_{s}\right)$ close to 5 .

\subsection{3 $F_{s}=1.11 F_{o}$}

Figure 4 shows the solution for $F_{s}=0.26\left(=1.11 F_{o}\right)$. The response of the upstream cylinder and the aerodynamic forces acting on it are quite similar to that observed in the earlier cases. The downstream cylinder exhibits low amplitude vibrations, primarily in the cross-flow direction. The dominant non dimensional frequency of cross-flow oscillations and the time variation of lift coefficient for the upstream cylinder is 0.242 . For the downstream cylinder the numbers are 0.244 and 0.106 , respectively. The increase in the lateral width of the wake as a result of the oscillations of the cylinders is observed for all the cases.

\subsection{4 $F_{s}=1.28 F_{o}$}

Shown in Figure 5 is the solution for $F_{s}=0.30\left(=1.28 F_{o}\right)$. Beats can be observed in the time histories corresponding to the first cylinder. During certain time intervals the upstream cylinder is associated with high value of mean drag and high amplitude of the unsteady component of the drag coefficient. At other times it behaves as a stationary cylinder. The in-line oscillations of the downstream cylinder are quite large and are comparable to the cross-flow vibrations. It has been observed that the large amplitude oscillations of the cylinder are accompanied with a reduction in the amplitude of the unsteady lift coefficient, thereby reflecting its self-limiting nature $[12,13]$. However, in the present case, since lock-in is not observed and the cylinder does not undergo sustained large amplitude oscillations, the lift coefficient shows large amplitude variations. Some of the frames in the flow pictures for this case indicate the possibility of competition between the symmetric and alternate modes of vortex shedding. Such phenomenon has been observed earlier in various studies $[16,17,18,13]$. The non dimensional value of the dominant frequency associated with the time variation of the lift-coefficient and cross-flow vibrations of the two cylinder is 0.175 .

Zdravkovich [1] reported results for flow induced oscillations of two interfering circular cylinders in various arrangements for $R e$ in the range of $1 \times 10^{4}$ to $8 \times 10^{4}$ using laboratory experiments. His results indicate that for cylinders arranged in tandem, and at maximum amplitudes of the vortex-shedding excitation, the upstream cylinder exhibits larger amplitude oscillations when $P / D=2.5$ and 7 . However, for $P / D=4.5$, the downstream cylinder undergoes larger amplitude vibrations. It is apparent that the oscillation amplitudes of the cylinders are quite sensitive to their relative arrangement. He also observed that the maximum oscillation amplitude of the cylinders arranged in tandem with $P / D=4.5$ is realized when $F_{s}=0.20$. The present computations seem to suggest the same behavior.

\subsection{Flow past two cylinders in staggered arrangement; $T / D=0.7$, $P / D=5.5$}

The staggered arrangement of cylinders is the one that is most likely to occur in an engineering situation, for example, in heat exchangers and other cooling systems involving tube bundles. Experimental investigations for such arrangements with stationary cylinders have been reported by various researchers in the past $[8,9,25]$. Numerical simulations of such flows for $R e=100$

and 1000 were reported by Mittal et al. [10] and for past periodic arrays of cylinders at $R e=100$ by Johnson et. al. [26]. 
The $R e=1000$ flow for this arrangement of stationary cylinders is quite different than the one at Reynolds number 100 [10]. The vortex shedding from the two cylinders is almost antiphase. The vortices that are shed from the upper and lower surfaces of the upstream cylinders pass below the lower surface of the downstream cylinder. Their interaction with the upper surface of the downstream cylinder is almost insignificant compared to that at $R e=100$. The vortex-shedding frequency for both the cylinders is $F_{o}=0.226$.

\subsection{1 $\quad F_{s}=F_{o}$}

Figure 6 shows the time histories of the lift and drag coefficients and the response of the cylinders for $F_{s}=0.226\left(=F_{o}\right)$ for the fully developed solution. Compared to the flow past cylinders in tandem arrangement, the flow for the staggered arrangement takes much longer to develop. The upstream cylinder behaves like a single cylinder and its response reaches an almost temporally periodic state. The downstream cylinder, however, exhibits lower amplitude oscillations and does not reach a temporally periodic state. The non dimensional frequency corresponding to the cross-flow oscillations of both the cylinders is 0.221 . The lift coefficient for the upstream cylinder varies with a dominant non dimensional frequency of 0.221 while the dominant frequency for the downstream cylinder is 0.167 .

Also shown in Figure 6 are the pressure and vorticity fields for the fully developed solution. Unlike the flow for stationary cylinders, the vortices shed from the upper surface of the first cylinder glide over the upper surface of the second cylinder. This is possible by the increased lateral width of the wake as a result of the oscillations of the first cylinder. The vortex shedding from the second cylinder is strongly influenced by the unsteady wake of the upstream cylinder. As a result of these oscillations, compared to the stationary cylinders, the mean value of the drag coefficient and the amplitude of its time varying component reduce significantly for the downstream cylinder. However, an increase in both the values is observed for the upstream cylinder.

\subsection{2 $\quad F_{s}=1.15 F_{o}$}

Figure 7 show the solution for $F_{s}=0.26\left(=1.15 F_{o}\right)$. The solution is very similar to the previous case $\left(F_{s}=F_{o}\right)$ except that, in the present case, the amplitude of the cylinder oscillations is lower and the Antisymmetrical mode $A-I V$ [18] (called as mode 2P by Williamson and Roshko [16]) of vortex shedding is more prominent for the upstream cylinder. Each cycle, in this mode of vortex shedding, is associated with two pairs of vortices being released from each of the upper and lower surfaces of the oscillating cylinder. The non dimensional frequency of the cross-flow oscillations of the two cylinders is 0.242 . The dominant frequency in the time-variation of the lift coefficient for the upstream cylinder is 0.242 while it is 0.097 for the downstream cylinder.

Zdravkovich [1] has reported results for flow induced oscillations of two interfering circular cylinders in a similar arrangement at higher Reynolds numbers. He reports that the maximum oscillation amplitude of the cylinders is observed for values of $F_{s}$ close to 0.21 . At these values of $F_{s}$, the amplitude of vibration of the upstream cylinder is larger than that of the downstream cylinder. The observations in the present study are consistent with these.

\section{Conclusions}

A numerical study has been carried out to study the flow-induced vibrations of a pair of cylinders in tandem and staggered arrangements at Re 1000. The cylinders are separated by 5.5 times the cylinder diameter in the streamwise direction. For the staggered arrangement, the 
spacing between the two cylinders is 0.7 times the cylinder diameter. As reported by researchers earlier, these arrangements of cylinders fall in the regime of wake interference. A summary of the results from the computations for various values of the non-dimensional structural frequency $\left(F_{s}\right)$ is shown in Figure 8. The downstream cylinder lies in the wake of the upstream cylinder and experiences wake-induced flutter. In almost all the cases the upstream cylinder responds like an isolated single cylinder. Soft lock-in is observed in many cases; the vortex-shedding frequency of the cylinders is detuned from the structural frequency. The trajectory of the upstream cylinder resembles a figure of 8 while it is quite disorganized for the downstream cylinder. In certain cases, the oscillations of the cylinders result in an alternate mode of vortex shedding. Vibration of cylinders is usually accompanied by an increase in drag. However, for the staggered arrangement and $F_{s}=F_{o}$ a reduction in the drag coefficient is observed as a result of the cylinder oscillations.

\section{Acknowledgment}

Partial support for this work has come from the Department of Science and Technology, India under the project number DST-AE-95279 with Department of Aerospace Engineering, IIT Kanpur.

\section{References}

[1] M.M. Zdravkovich. Flow induced oscillations of two interfering circular cylinders. Journal of Sound and Vibration, 101(4):511-521, 1985.

[2] J.A. Jendrzejczyk, S.S. Chen, and M.W. Wambsganss. Dynamic response of a pair of circular tubes subjected to liquid cross flow. Journal of Sound and Vibration, 67:263-273, 1979 .

[3] R. King and D.J. Johns. Wake interaction experiments with two flexible cirular cylinders in flowing water. Journal of Sound and Vibration, 45:259-283, 1976.

[4] Y.T. Tsui. On wake-indiced flutter of a circular cylinder in the wake of another. Journal of Applied Mechanics, Transactions of the ASME, 99:194-200, 1977.

[5] A. Bokaian and F. Geeola. Wake-induced galloping of two interfering circular cylinders. Journal of Fluid Mechanics, 146:383-415, 1984.

[6] C.W. Knisely and M. Kawagoe. Force-displacement measurements on closely spaced tandem cylinders. In M. Ito, M. Matsumoto, and N. Shiraishi, editors, Bluff Body Aerodynamics and its Applications, pages 81-90. Elsevier, Amsterdam, 1990.

[7] M. Matsumoto, N. Shiraishi, and H. Shirato. Aerodynamic instabilities of twin circular cylinders. In M. Ito, M. Matsumoto, and N. Shiraishi, editors, Bluff Body Aerodynamics and its Applications, pages 91-100. Elsevier, Amsterdam, 1990.

[8] S. S. Chen. Flow-Induced Vibrations of Circular Cylindrical Structures. Hemisphere Publishing Corporation, New York, 1987.

[9] M.M. Zdravkovich. Review of flow interference between two circular cylinders in various arrangements. Journal of Fluids Engineering, Transactions of the ASME, 99:618-633, 1977. 
[10] S. Mittal, V. Kumar, and A. Raghuvanshi. Unsteady incompressible flow past two cylinders in tandem and staggered arrangements. International Journal for Numerical Methods in Fluids, 25:1315-1344, 1997.

[11] S. Mittal and T.E. Tezduyar. A finite element study of incompressible flows past oscillating cylinders and airfoils. International Journal for Numerical Methods in Fluids, 15:10731118, 1992.

[12] S. Mittal and V. Kumar. Finite element study of vortex-induced cross-flow and in-line oscillations of a circular cylinder at low Reynolds numbers. International Journal for Numerical Methods in Fluids, 31:1087-1120, 1999.

[13] S. Mittal and V. Kumar. Flow induced vibrations of a light circular cylinder at Reynolds numbers $10^{3}$ to $10^{4}$. submitted to Journal of Sound and Vibration, 2000.

[14] G.H. Koopmann. The vortex wakes of vibrating cylinders at low Reynolds numbers. Journal of Fluid Mechanics, 28:501-512, 1967.

[15] T. Sarpkaya. Vortex-induced oscillations: A selective review. Journal of Applied Mechanics, 46:241-258, 1979.

[16] C.H.K. Williamson and A. Roshko. Vortex formation in the wake of an oscillating cylinder. Journal of Fluids and Structures, 2:355-381, 1988.

[17] A. Ongoren and D. Rockwell. Flow structure from an oscillating cylinder Part 1. Mechanisms of phase shift and recovery in the near wake. Journal of Fluid Mechanics, 191:197223, 1988.

[18] A. Ongoren and D. Rockwell. Flow structure from an oscillating cylinder Part 2. Mode competition in the near wake. Journal of Fluid Mechanics, 191:225-245, 1988.

[19] S. Mittal and V. Kumar. Flow-induced oscillations of two cylinders in tandem and staggered arrangement. to appear inJournal of Fluids and Structures, 2000.

[20] T.E. Tezduyar, M. Behr, and J. Liou. A new strategy for finite element computations involving moving boundaries and interfaces - the deforming-spatial-domain/space-time procedure: I. The concept and the preliminary tests. Computer Methods in Applied Mechanics and Engineering, 94(3):339-351, 1992.

[21] T.E. Tezduyar, M. Behr, S. Mittal, and J. Liou. A new strategy for finite element computations involving moving boundaries and interfaces - the deforming-spatial-domain/spacetime procedure: II. Computation of free-surface flows, two-liquid flows, and flows with drifting cylinders. Computer Methods in Applied Mechanics and Engineering, 94(3):353$371,1992$.

[22] Y. Saad and M. Schultz. GMRES: A generalized minimal residual algorithm for solving nonsymmetric linear systems. SIAM Journal of Scientific and Statistical Computing, 7:856869, 1986.

[23] T.E. Tezduyar, S. Mittal, S.E. Ray, and R. Shih. Incompressible flow computations with stabilized bilinear and linear equal-order-interpolation velocity-pressure elements. Computer Methods in Applied Mechanics and Engineering, 95:221-242, 1992. 
[24] C.H.K. Williamson. Vortex dynamics in the cylinder wake. Annual Review of Fluid Mechanics, 28:477-539, 1996.

[25] M. Kiya, M. Arie, H. Tamura, and H. Mori. Vortex shedding from two circular cylinders in staggered arrangement. Journal of Fluids Engineering, Transactions of the ASME, 102:166-173, 1980.

[26] A.A. Johnson, T.E. Tezduyar, and J. Liou. Numerical simulation of flows past periodic arrays of cylinders. Computational Mechanics, 11:371-383, 1993. 

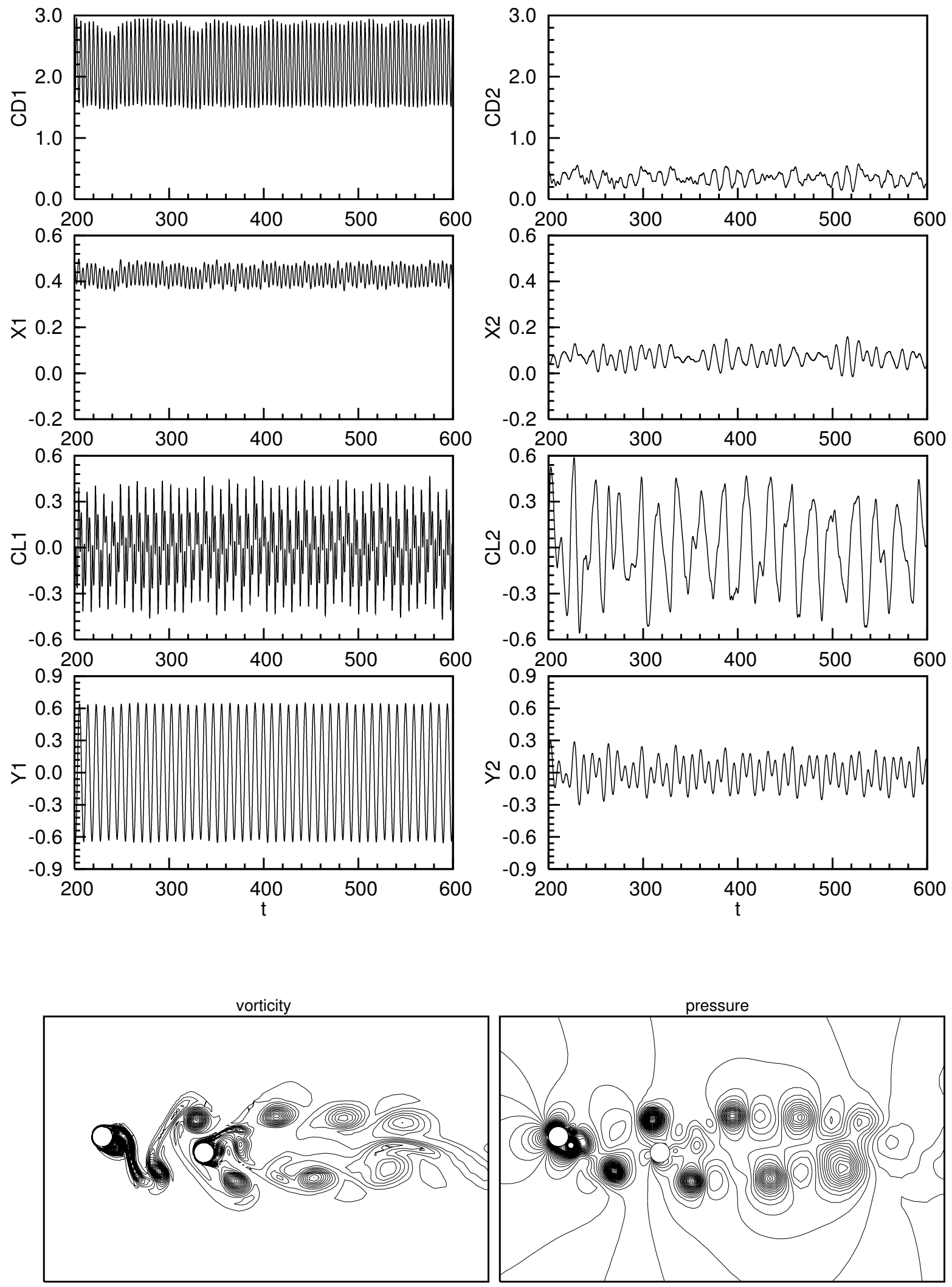

Figure 2: $R e=1000$ flow past two oscillating cylinders with $F_{s}=0.234\left(=F_{o}\right)$ in tandem arrangement $T / D=0, P / D=5.5$ : time histories of the drag and lift coefficients and response of the cylinders and flow at $t=375.45$. 

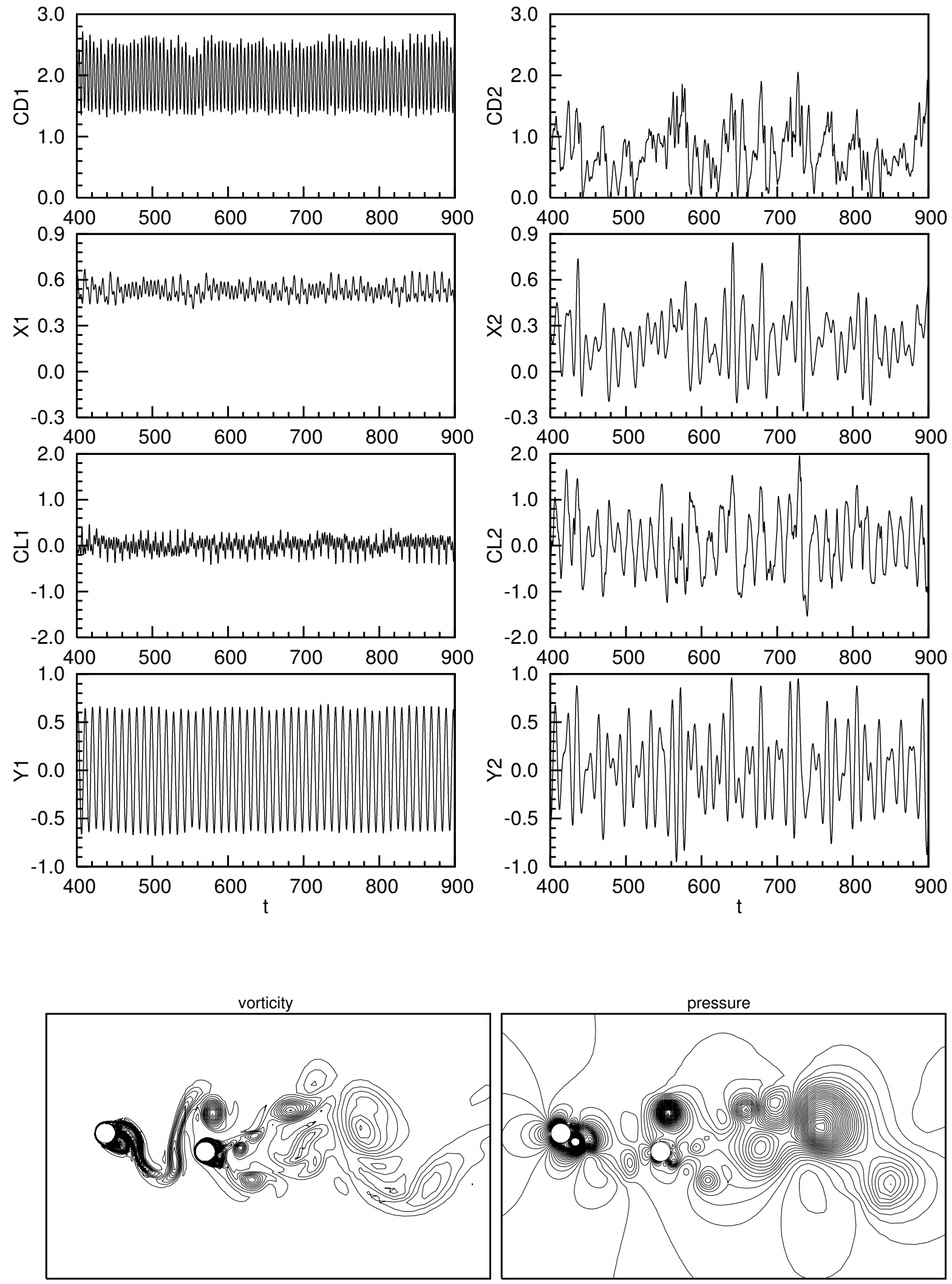

Figure 3: $R e=1000$ flow past two oscillating cylinders with $F_{s}=0.20\left(=0.85 F_{o}\right)$ in tandem arrangement $T / D=0, P / D=5.5$ : time histories of the drag and lift coefficients and response of the cylinders and flow at $t=861.75$. 

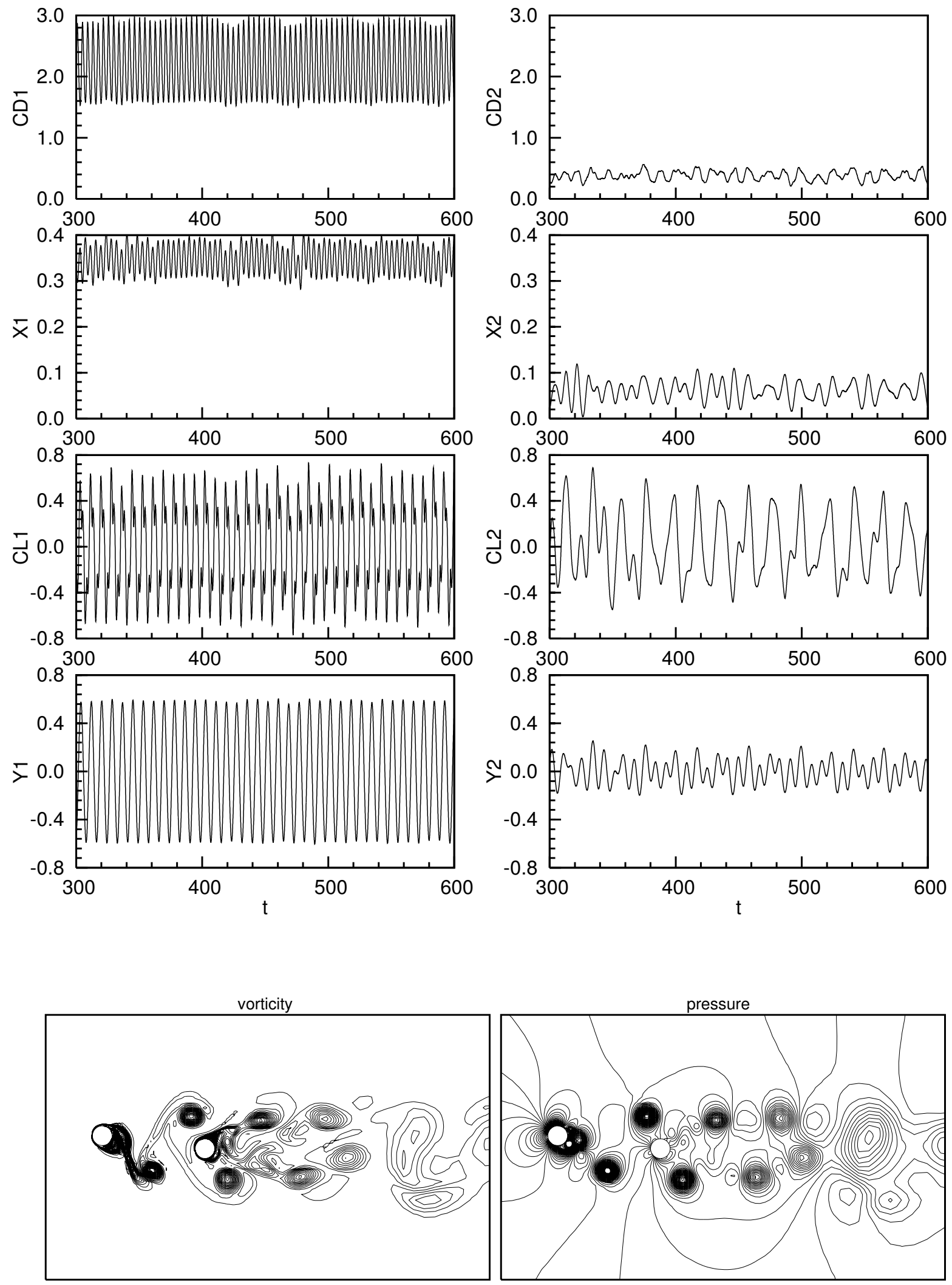

Figure 4: $R e=1000$ flow past two oscillating cylinders with $F_{s}=0.26\left(=1.11 F_{o}\right)$ in tandem arrangement $T / D=0, P / D=5.5$ : time histories of the drag and lift coefficients and response of the cylinders and flow at $t=528.55$. 

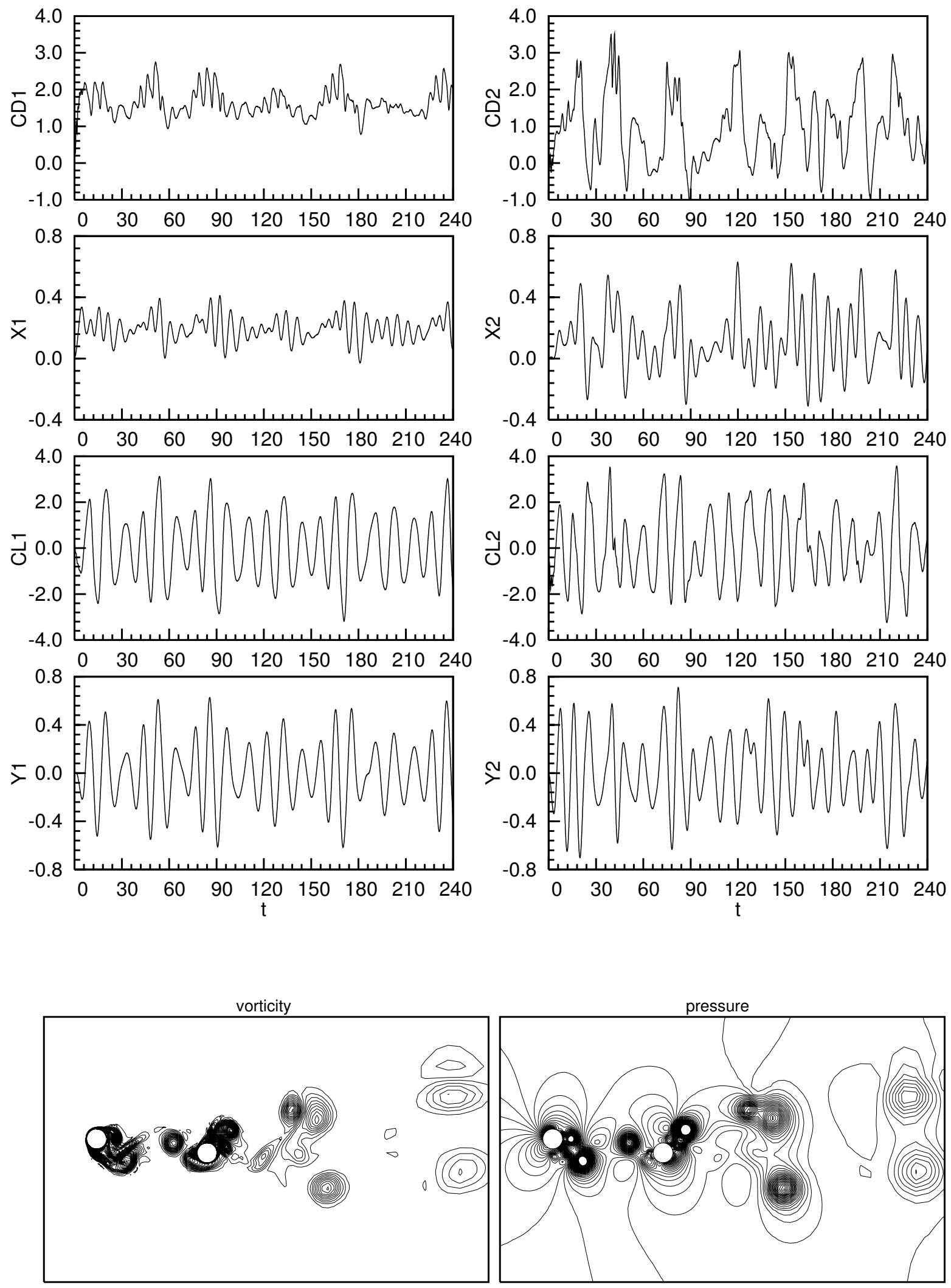

Figure 5: $R e=1000$ flow past two oscillating cylinders with $F_{s}=0.30\left(=1.28 F_{o}\right)$ in tandem arrangement $T / D=0, P / D=5.5$ : time histories of the drag and lift coefficients and response of the cylinders and flow flow at $t=178.55$. 

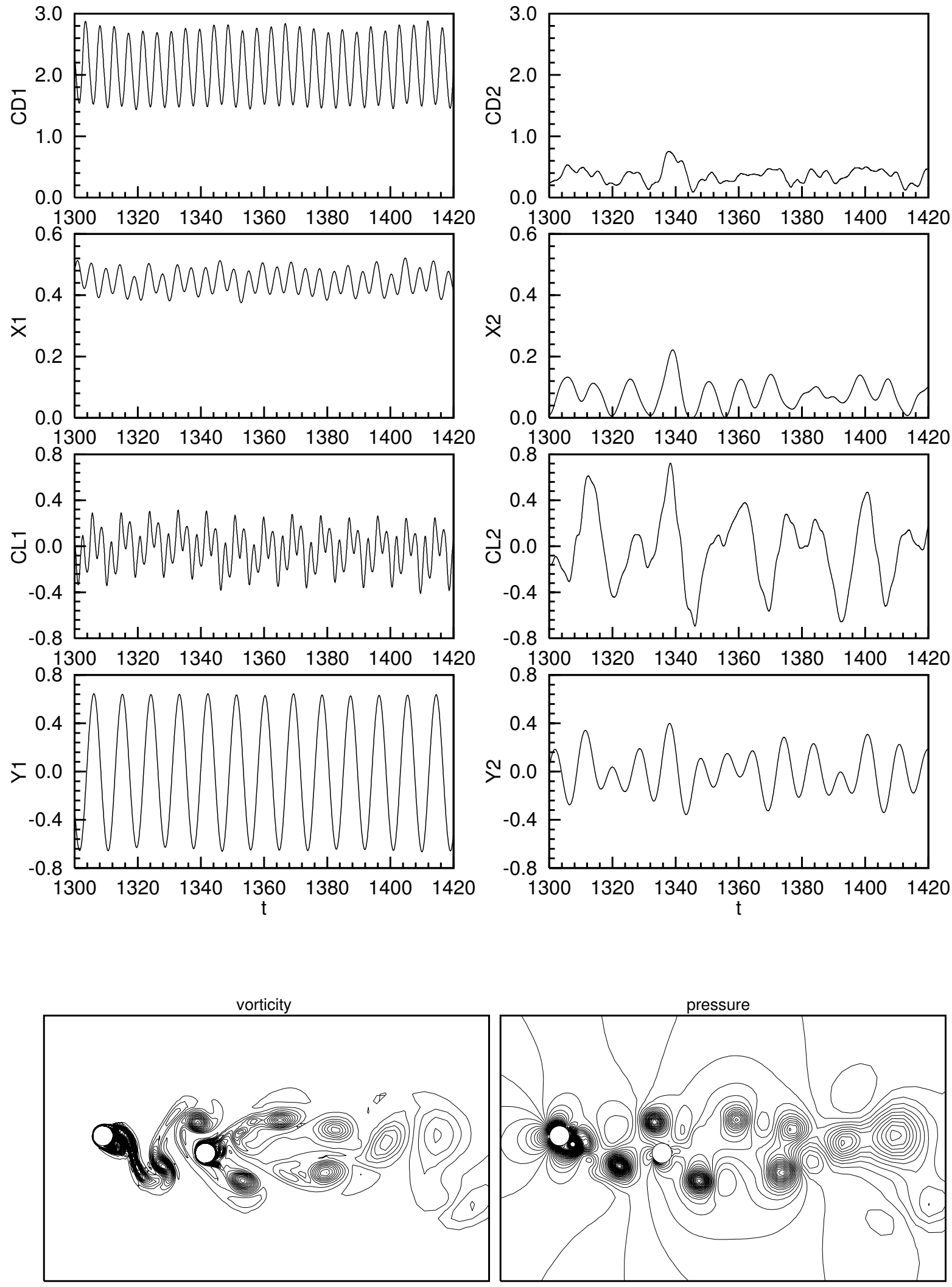

Figure 6: $R e=1000$ flow past two oscillating cylinders with $F_{s}=0.226\left(=F_{o}\right)$ in staggered arrangement $T / D=0.7, P / D=5.5$ : time histories of the drag and lift coefficients and response of the cylinders and flow at $t=1433.70$. 

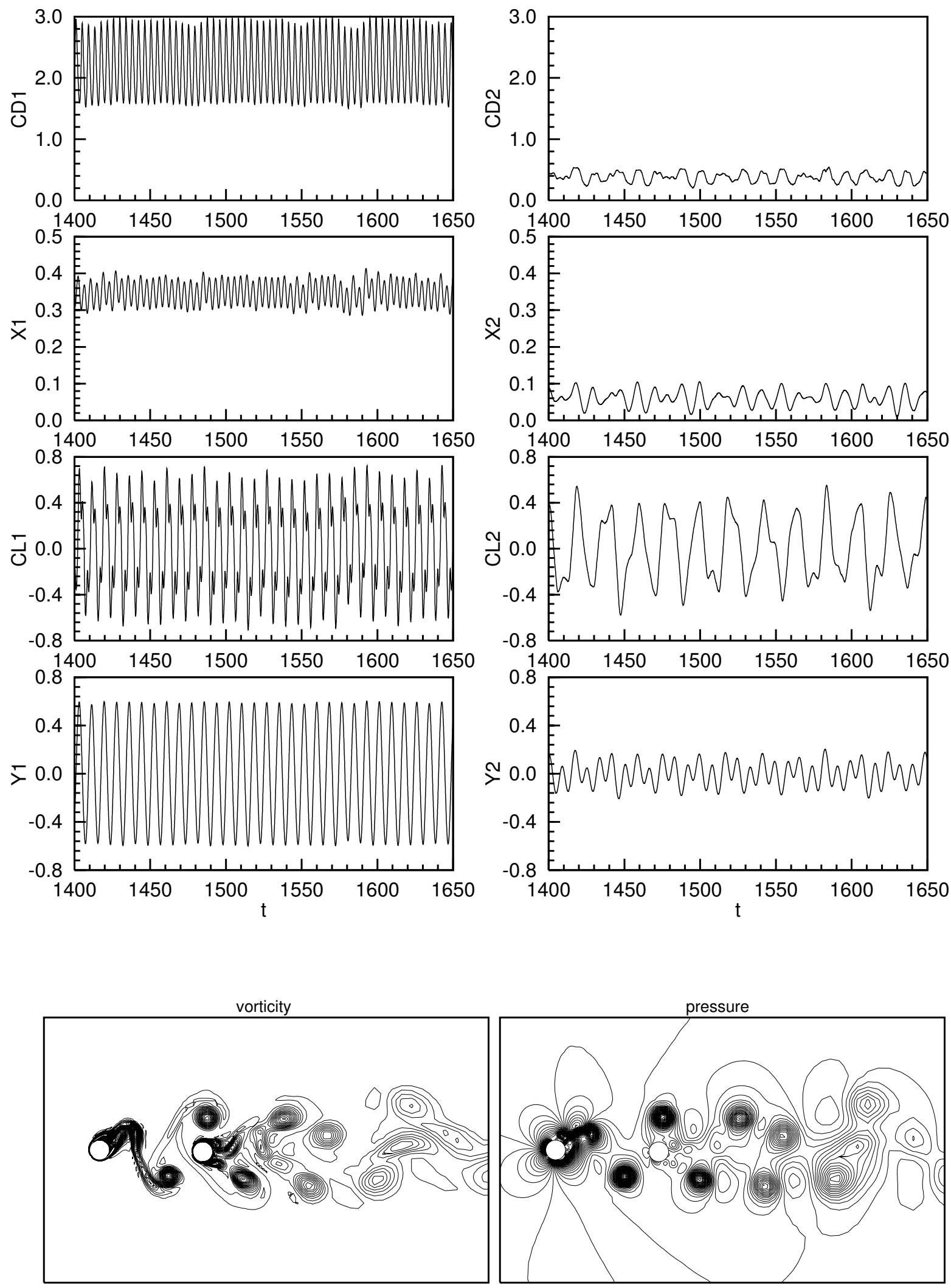

Figure 7: $R e=1000$ flow past two oscillating cylinders with $F_{s}=0.26\left(=1.15 F_{o}\right)$ in staggered arrangement $T / D=0.7, P / D=5.5$ : time histories of the drag and lift coefficients and response of the cylinders and flow at $t=1686.70$. 

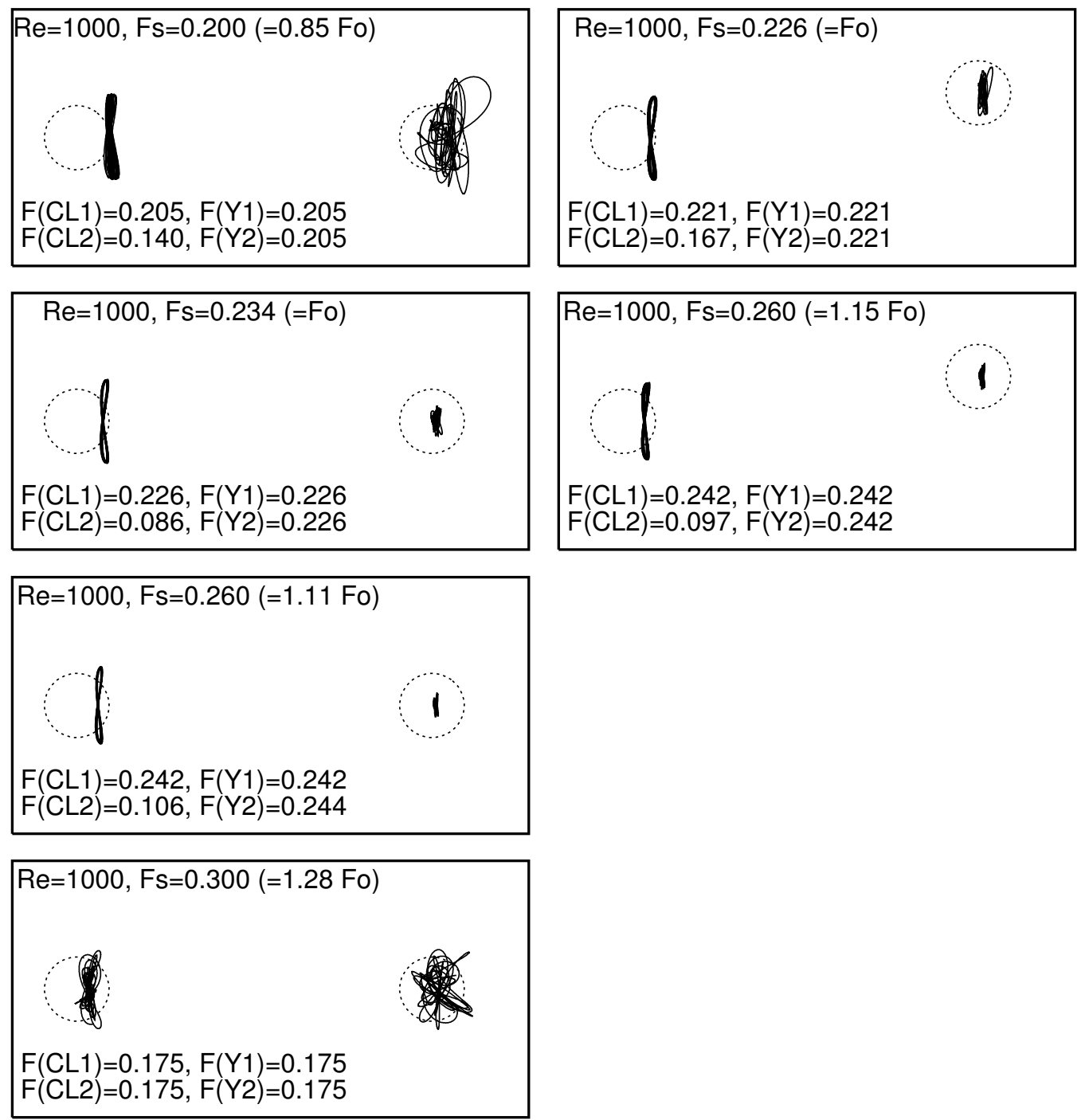

Figure 8: Summary of the flow past two oscillating cylinders. Results for cylinders in tandem $(P / D=5.5, T / D=0)$ are on the left and those for the staggered arrangement $(P / D=$ $5.5, T / D=0.7)$ are on the right. The trajectory of the center of the cylinders is shown in solid lines while the initial location of the cylinders is shown in dotted lines. Also shown are the dominant frequencies for the time-variation of the lift coefficients and the cross-flow oscillations of the two cylinders. 